9. Ibid., p. 603 .

10. J.K. Galbraith, La cultura de la satisfacción, Barcelona, Ariel, 1992, p. 26.

11. Ibid, p. 29.

12. Ibiat, p. 30 .

13. Tbid, p. 32.

14. N. Redondo, El sindicalismo de clase en al moviniento obrera, Madrid, Escuela Julián Besteiro, 1993, p. 42.

15. $M b i d, 43$.

16. M. Albert, Capitalismo contra capitalisno, Barcelona, Paidós, 1991, p. 110.

17. VV.AA., La larga noche neoliberal, Barcelona, Icaria-Instituto sindical de estudios, 1993.

\title{
«Dígales que mi vida ha sido maravillosa»: ética y existencia en $\mathbf{L}$. Wittgenstein
}

\author{
ANTONI DEFEZ I MARTÍN \\ Universidad de Valencia
}

\begin{abstract}
Mi ideal es una cierta indiferencia: un templo que cierre el paso a las pasiones sin ser afectado por ellas.
\end{abstract}

L. WITTGENSTEIN, Vemntschte Benterkungen

Una de las constantes del pensamiento de Wittgenstein es su concepcion unocientificistan de la actividad filosófica: la filosofía siempre debía quedar al margen de la ciencia natural. $\mathrm{Y}$ ello a pesar de que, en su opinión, el conocimiento científico era la mejor forma de acceder al "cómo" de la realidad. Recordemos que para el Tractatus (TLP) el ámbito del usentido» coincidía con el ámbito del conocimiento científico (p.e., $T L P, 4.11$ y 6.53), mientras que el segundo Wittgenstein reconocerá la superioridad de la ciencia respecto a otras "formas de vida" $\mathbf{u}$ otros "sistemas de creencias», sin ser, no obstante, justificable esta superioridad ni filosófica ni moralmente -digamos, "racionalmente" (vid., p.e., Observaciones sobre La Rama Dorada de Frazer). Ahora bien, y pese a estos meritos, para Wittgenstein, la filosofía no estaba interesada por lo que pudiera establecer la ciencia. Los logros de ésta no podían ser usados para solucionar los problemas de aquélla; y tampoco era tarea de la filosofia ofrecer una reconstrucción o una fundamentación del conocimiento científico. Por el contrario, la actividad filosofica debía reducirse a actividad o análisis dilucidatorio del lenguaje, bien en el sentido de esclarecer sus condiciones trascendentales de posibilidad a partir de la lógica extensional en la epoca del Tractatus, o bien en la más modesta función, ya dentro del pensamiento que se desarrolla en las Investigaciones Filosoficas (IF), de hacer patentes las condiciones que de facto posibilitan y regulan los distintos «juegos lingüísticos». Entendida de esta manera, la filosofía "había de dejar todo como están, ya que a través de su ejercicio, al no lanzar hipótesis y al no utilizar las hipótesis de la ciencia, no consigue ningún conocimiento nuevo que antes no poseyéramos (vid., p.e., IF $\$ 122-128, \S 309$ y $\S 599$ ). En realidad, la única ganancia que con ella alcanzaríamos es la uclaridad conceptual» $y$, en consecuencia, el benefi- 
cio de disolver o de hacer desaparecer los problemas filosóficos que nos atormentan, problemas que surgirfan de una mala comprensión de la lógica que gobierna nuestras palabras.

Teniendo presente esta concepción del status y de la función de la filosofia y su relación con el ámbito de lo científico, me gustaría, sin entrar como ha hecho W. Warren Bartley III en el terreno de las motivaciones personales inconfesables de Wittgenstein, ${ }^{1}$ analizar en el presente escrito cómo tales posicionamientos se corresponden con su planteamiento existencial y ético. Esta, evidentemente, no es una aproximación demasiado frecuentada dentro de la exégesis que ofrecen los círculos analíticos. No obstante, el planteamiento existencial y ético de un filosofo es también una tesis filosofica. $Y$, además, en el caso de Wittgensteín, una tesis reconocida por el mismo. Recordemos, por ejemplo, las palabras que dirigió a P. Engelman refiriéndose al Tractatus: «El punto central del libro es ético [...]. Mi trabajo consta de dos partes: la expuesta más todo lo que no he escrito. $Y$ es precisamente esta segunda parte la más importantem.2 Por tanto, no olvidar este tipo de motivaciones, como correctamente señalaron A. Janik y $S$. Toulmin ${ }^{3}$ al comienzo de los años setenta, puede reportarnos alguna luz sobre las intenciones de Wittgenstein.

***

Ex hypothesi, consideraré que a Wittgenstein, como a la mayoría de los filosofos de nuestra tradicion, le preocupaba, en tanto que problema existencialmente básico, la relación "yo-mundo" 0 , si se quiere, el problema de la relación entre lo subjetivo y lo objetivo o entre lo privado y lo público. Respecto a este núcleo de problemas, y a pe- sar de la dificultad que produce el hecho de que Wittgenstein ni fue un pensador de una unica filosofía ni un filosofo de dos filosofías in toto antitéticas, creo que podemos presentar su posición mediante las siguientes tesis:

I) La admiración ante el carácter insólito de lo que está dado o es cotidiano, esto es, la admiración ante el yo y el mundo.

II) La transparencia de lo que hay o de lo que está dado, que es el yo y el mindo.

III) La necesidad de hacer que lo insólito devenga sólito o familiar a través del esfuerzo filosofico de hacerlo transparente.

rv) La insuficiencia de la transparencia de lo dado y de este hacer patente la transparencia de lo dado.

Estas tesis tienen un alcance y una significación diferentes. $\Lambda$ sí, (I), (II) y (III) podrian hacer de Wittgenstein un pensador, digamos, empirista (en el sentido de que idealizadamente hay un posicionamiento denominado «empirismon) o, si se quiere, lo que W. James bautizó como una "mente ruda" en oposicion a las ementes delicadas». 4 En concreto, Wittgenstein asume una actitud abiertamente antiplatónica. Como escribe él mismo en Philosophical Grammar ( $P G)$, "nada esta escondido [...], nada está oculto" ( $P G$, $\S 63)$; no hay ningún topos ouranus que filosóficamente podamos hacer servir; o, por decirlo de otra manera: la solución a nuestros problemas no se encuentra fuera de la caverna. Por el contrario, la transparencia que persigue la filosofía consiste simplemente en hacer patente lo que ya tenemos ante nosotros pero que olvidamos frecuentemente: el efectivo funcionamiento de nuestro lenguaje.

Sin embargo (rv) parece apuntar en otra dirección: expresaria algo que históricamente tiene santo y seña y que el 
empirismo y las umentes rudas", al menos en apariencia, no han tematizado suficientemente, a saber, la "tendencia natural a la metafísican. Esta tesis (V), con todo, exige una tesis (v) que resuelva la contradicción que parece mantener con las tres primeras. Echando mano de un verso de LaoTsé, padriamos anunciarla diciendo: «lo no existente es capaz de penetrar lo impenetrablex.s Dicho de otra forma: lo que no está en el mundo ni es un posible objeto de discurso es capaz de penetrar y alterar el mundo. Llamaré a esta tesis la tesis ética de Wittgenstein.

Pero no acaba aquí la explicación. La argumentación de Wittgenstein, si ha de tener alguna fuerza, tiene que descansar en alguna otra tesis presupuesta que debemos sacar a la luz. Siguiendo la broma de F.P. Ramsey, podríamos decir que si Wittgenstein quiere tener derecho a «silbar» (o "mostrar") lo que no se puede "decir", debe tener alguna justificación que a ello le autorice. Para ver cuál pueda ser ésta, consideremos en qué sentido fue Wittgenstein un «antimetafísicos y por qué lo fue. De entrada, es necesario enfatizar que alguien puede declararse "antimetafísico" por dos razones distintas: bien porque la metafísica carece de sentido y es una «insensatezn, o bien porque aquello que cl metafísico "pretende afirmar" carece de sentido y es una "insensatez". A los ojos de Wittgenstein existe aqur una diferencia importante: la primera implica la segunda, pero no al reves. Para Wittgenstein, lo metafísico - «lo místico» (das Mystische) - existe en tanto que actitud $\mathrm{y}$ tendencia humana, pero no puede existir como discurso o como conocimiento (vid., p.e., TLP, 6.53). Ahora bien, esto no parece suficiente. Podriamos preguntarnos aún en qué descansa la tesis (rv) y por qué Witt- genstein se nos presenta como un empirista inconsecuente, ya que apostando por la tesis (IV) no renuncia a las tesis (I), (II) y (III).

El problema parece radicar en la importancia o relieve filosófico que se quiera otorgar a lo que enuncia la tesis (Iv). Y ello en función de la concepción abiertamente reconocida por Wittgenstein de que no existe, y si existe carece de interés y de relevancia filosófica, la posibilidad de eexplicar», a partir de los conocimientos que poseemos sobre la realidad y nosotros mismos, esta tendencia hacia la metafísica. A diferencia de lo que pensaron los miembros del Círculo de Viena y, en concreto, a diferencia de la concepción carnapiana del metafísico como "músico frustradon," para Wittgenstein (IV) está legitimada filosóficamente y existencialmente porque expresa un whecho bruton inanalizable e inexplicable, esto es, un punto de partida o fundamento del cual no se puede dar razón y sobre el cual hay que observar la política del noli me tangere. Llamemos a esta presuposición la tesis (VI).

Parece claro que aquí nos las vemos con la concepción wittgensteiniana del conocimiento cientifico. En su opinión, habria dos maneras excluyentes de considerar lo cientifico: una que critica - la que podriamos llamar la visión "optimistas - y otra que defiende - la denominaremos la visión "pesimista". Wittgenstein se desmarca de la "ilusion" que se produce cuando aceptamos que el conocimiento científico lo puede explicar todo o que puede servir como tribuna de justificación y de crítica racional de todo -incluso del ámbito de lo humano y en su seno de cosas como la expresada por (IV). A pesar de reconocer, como ya hemos indicado, los indiscutibles méritos de la ciencia, Wittgenstein no acepta que con la ciencia o que con el tipo de ex- 
plicaciones que ésta ofrece se acabe todo lo que tiene importancia, o que con la ciencia se acabe el misterio. En realidad, en su opinion, to que es realmente relevante desde el punto de vista humano empieza a partir o queda más allá de donde puede llegar la ciencia (vid., p.e., $T L P, 6.371,6.372$ y 6.52), Es por esta razón por la que el optimista vive bajo una "ilusión". $\mathrm{Y}$ es también la razón por la que Wittgenstein, como podemos comprobar a lo largo del conjunto de notas recopilado por G.H. von Wright bajo el nombre de Vermischte Bemerkungen, no oculto su desinterés y disgusto hacia la cultura y la sociedad cientificista y tecnificada en que vivió.

Esta manera de presentar las cosas, no obstante, parece ser el resultado de un falso dilema, porque entre las dos opciones expuestas se supone que ha de darse una especie de disyuncion exclusiva cuando, de hecho, no tiene por qué ser asi. Por el contrario, se puede hacer gala de un temperamento, digamos, "tranquilo". No se trata de ser optimista o pesimista: se puede aceptar que la ciencia no lo explica todo y que es posible que nunca lo consiga - es decir, aceptar que la ciencia no acaba con el misterio- $y$, sin embargo, reconocer que el conocimiento científico o positivo es la mejor cosa de que disponemos a la hora de comprender el mundo y a nosotros mismos. No fue ésta, sin embargo, la actitud de Wittgenstein; la suya viene mejor expresada, como hemos indicado antes, por el pesimismo o el escepticismo existencial hacia lo científico y, como ya se dijo al principio, este tipo de desconfianza fue una de las constantes que siempre configuró la mancra cómo entendio la actividad filosófica.

Ahora bien, el cuadro todavía no está completo. Hemos visto la significación y el papel que juega la tesis (IV), pero falta por ver aún por qué existe una tesis como (IV) y una tesis como (v), es decir, la tesis de la tendencia hacia la metafísica y la tesis ética. La solución, dentro de la argumentación de Wittgenstein, seria la sigulente: la tendencia hacia la metafísica y la ética hacen su irrupción en el mundo a través de la existencia del sujeto. Sea ésta la tesis (VI), tesis que en manos de Wittgenstein, y de acuerdo con lo que enuncia la tesis (v), necesita de la siguiente matizacion: lo que importa de la existencia del sujeto a la tendencia hacia la metafísica es algo que el discurso positivo o las explicaciones cientificas han de dejar intocado. Como ha señalado P.F. Strawson en Individuals, Wittgenstein elabor 6 una doctrina sin sujeto* del yo, en la medida en que apuesta por una especie de malabarismo que deja al sujeto en penumbra u oculto. ${ }^{7} Y$ ello no solo como consecuencia de la tesis (vi), sino también como aquello que es necesario para afirmar las tesis (IV) y (v).

Efectivamente, en el Tractatus el sujeto queda fuera del mundo. El sujeto que "piensa" y que se representa estados de cosas -el sujeto que uproyecta" el lenguaje sobre la realidad-y que es también el sujeto que "quiere» en sentido ético, "no existe", se encuentra más allá de los límites del lenguaje, más allá de lo que es verdaderofalso y, por tanto, más allá de las explicaciones cientificas. Se trata del esujeto trascendentaly. A su vez, para el segundo Wittgenstein tampoco hay posibilidad de usar explicaciones causales $-y$, entre ellas, las explicaciones de la ciencia- para explicar o cvaluar racionalmente la conducta lingüística $y$ epistémica de los hombres. Filosóficamente, lo que podemos alcanzar es una mera «descripción», tanto de los usos lingüisticos como del papel y del status de las creencias de los hombres 
dentro de sus prácticas cognoscitivas. Nada más sería necesario, en opinión de Wittgenstein.

Así las cosas, lo que parece ser la intención de Wittgenstein es, en resumen, que la irrupción del sujeto en el mundo y la tendencia hacia la metafísica son "hechos brutos", puntos de partida inanalizables, de los cuales no podemos dar ninguna explicación, pero que use muestran" en el uso del lenguaje. $Y$ es precisamente este carácter de «punto de partida inanalizable» lo que permitira su relevancia filosofi$c a$, que queda plasmada en las tesis (Iv) y (v). Pero, ¿de que punto de partida se trata? De forma evidente (IV) y (v) no son un punto de partida en el sentido de un fundamentum inconsussum veritatis: no reportan ni amplian nuestro conocimiento de la realidad; tampoco lo hacen más cierto ni evidente. Podríamos considerarlos como fundamentum inconsussum, pero no de la verdad, sino del ethos que hay que observar ante la vida y el mundo. Nada existe detrás del mundo, ni detrás de los objetos ni de los hechos, ni más allá de la lógica o de la gramática $y$, sin embargo, lo dado y su transparencia nos sabe a poco; por eso tendemos a ir más allá, tendemos a correr contra los límites. Podriamos decir que para Wittgenstein lo que nos define a los hombres es nuestro carácter fronterizo y disidente con respecto a lo dado.

"Lo místico", pese a existir, no sirve cognoscitivamente de ninguna ayuda, ni siquiera es lingüisticamente expresable. Consiste en una manera, digamos, "oblicuas de contemplar lo real y conduce simplemente a una actitud ética y estética ante la vida y el mundo. Esta actitud tampoco será lingüísticamente expresable, no es conocimiento ni es capaz de reportarlo. Con otras palabras: "lo místico", que es la manera filosoficamente correcta de ver, se resuelve en la manera filosoficamente correcta de estar aqui, de vivir. Podernos caracterizarla de la siguiente forma: (I) la contemplación de la existencia del mundo como algo que carece de explicacion y de sentido, esto es, como un simple "milagro estéticon; (iI) la visión del mundo como una totalidad ordenada lógica o gramaticalmente que no remite a nada distinto de sí misma; (III) darse cuenta de que con lo que se ajusta a las condiciones del «decir" y del "conocer» se acaba todo lo que podemos decir y conocer; y (IV) que con la mera "vivencia" del milagro estético de la existencia del mundo -de los límites y de la transparencia de lo dado-, y con el «silencio» resultante de la reducción filosófica, se han solucionado, disuelto o desvanecido todos los problemas importantes, los problemas vitales y existenciales.

Vemos, pues, que "lo místico" nos lleva a ulo ético». Y «lo ético», para Wittgenstein, se resuelve en una simple uactitud" ante el mundo y la vida. Como podemos comprobar en las últimas páginas del Tractatus y en las notas redactadas entre junio y agosto de 1916 y que vienen recogidas en sus Notebooks, la propuesta de Wittgenstein recuerda las antiguas éticas helenísticas del ideal de vida del sabio: persigue individualmente la eudaimonia. la cual se legitima ante la vida de una forma casi tautológica, ya que lo consustancial a vivir es la "vida feliz». A su vez, el carácter individual de la felicidad viene exigido no sólo porque la "vida feliz" es cosa del sujeto sino, además, por la misma manera como se ha de entender y se puede alcanzar: mezcla de sabiduría - vida entregada a la contemplación teorética- y de ascesis vital por parte del individuo.

Pero hagamos una advertencia antes de continuar: el cómo y el qué de la "vida fcliz" no han de ser entendidos 
en terminos de causa-efecto. Eso haria de la actitud ética una actividad que tiene incidencia sobre los hechos del mundo, cosa imposible para Wittgenstein, ya que en ese caso la actitud ética sería un fenómeno descriptible, cognoscible, analizable y evaluable. Sería más correcto afirmar que en la actitud etica, en la medida en que ésta no consiste en una acción sobre el mundo, el qué y el cómo coinciden. Y coinciden en tanto que son y buscan una cindiferencia" o una uimperturbabilidad" ante los hechos - conceptos que evocan bien a las claras la «apatias estoica - la "ataraxia» epicúrea. No se trata de cambiar los hechos sino de alterar los "límites" del mundo.

Wittgenstein, sin embargo, con esta alteración de «límites» no está apostando por alguna posibilidad teorica de ir más alla de los límites del sentido y del conocimiento mediante la ética. No. Wittgenstein no es Kant; para ell lo pensable, lo cognoscible, y lo expresable significativamente coinciden estrictamente y los límites de uno son los límites de los otros. En realidad, esta alteración de límites consiste y se obtiene por la tranquilidad o la serenidad existencial que radica en la uno-acción», el no descar, la renuncia, la mera contemplación o la indiferencia ante lo que es dado. Más explícitamente: por la concordancia entre la voluntad y el mundo, en la medida en que el sujeto se niega a intervenir o a cambiar los hechos (vid, p.e, TLP, 6.423, 6.43). En eso parece consistir la visión del mundo sub specie aeternitatis de que nos habla el Tractatus.

De esta forma, la alteración de los limites del mundo mediante la actitud ética no consiste sino en la manera como vivenciamos la existencia de lo dado, en la manera como aquello que no existe o que no puede ser dicho significativamente que existe -a saber, el yo y la voluntad entendidos como actividad no fenoménica y no psicológica- penetra y altera aquello que, en rigor, no puede penetrar ni alterar. $\mathrm{He}$ aquí por qué anteriormente haclamos referencia al verso de Lao-Tsé. Tanto en la "vida feliz" como en la vida infeliz lo dado es, digamos, extensionalmente equivalente: no hay más realidad en una que en la otra. La diferencia, no obstante, descansa en la manera o en la actitud con que es vivida esa realidad. $Y$ esa actitud puede ser la actitud de aquel que se conforma, acepta y desea lo dado, reconciliandose así con el mundo, o la del que se rebela. Así el primero es el hombre sabio y feliz, mientras que el segundo es el desventurado y el vitalmente ignorante.

Esta propuesta ética se ve reforzada por las razones que, según Wittgenstein, no permitirían que la ética se constituyese como una disciplina significativa. Si tenemos presente lo que dijo en su Conferencia sobre ética (1929-1930) y en las conversaciones que mantuvo con los miembros del Círculo de Viena entre 1929 y 1932 sobre aquello que debería ser la ética, vemos que lo que tenía en mente era la idea de la ética como discurso que proporcionase justificaciones absolutas sobre lo bueno o, como él mismo los califica, «juicios de valor absoluto", esto es, una ética entendida como "reina filos6fica». ${ }^{\circ}$ Y claro, como tal cosa no es posible, por no ajustarse ningún juicio de este tipo a los límites del sentido fijados, la ética representaba un imposible, mejor, una insensatez. Tirando, asf, la cuerda tras el caldero, Wittgenstein nos ofrece su versión de la «falacia naturalista»: no es posible obtener o llegar a ningún «deber ser» porque todo aquello significativamente expresable o inteligible hace referencia simplemente al ser, esto es, se agota en la mera descripción de lo que hay. De 
hecho, todos los juicios de valor relativo -que no son juicios éticos para Wittgenstein - podrán reducirse a simples descripciones.

Ahora bien, no era necesario tirar la cuerda. Como sugirió Ferrater Mora en De la materia a la razón. únicamente partiendo de la presuposición de que la ética, si ha de ser alguna cosa, ha de ser una "reina filosófica" que proporcione "superjustificaciones", se puede, ante la imposibilidad de ver cumplido este ideal, proponer su disolución o una ética individualista de renuncia, aceptación y contemplación. ${ }^{9}$ Si Wittgenstein hubiese observado una actitud más favorable hacia la utilidad filosófica de los conocimientos positivos o cientílicos, podría haber aceptado la posibilidad de una ética basada en lo que sabemos de nosotros mismos $y$ de la realidad que nos circunda, una ética que hiciese utso de los valores morales, que como simples logros históricos y sociales favorecen la felicidad de los hombres. En este caso, evidentemente, la ética ya no podría ser una "reina filosofica", pero tampoco parece que haya necesidad de tal cosa: la ética, por ser empírica y tentativa, no tiene por qué ser menos valiosa. Con todo, como ya se ha reflejado, no fue ésta su posición: la filosofía no estaba necesitada de las hipótesis ni de las explicaciones positivas.

Volvamos, sin embargo, al carácter «individual" y "trascendental" de la ética del Tractatus. Estos rasgos pueden hacernos entender la instancia desde la cual fue escrita esta obra y también pueden ofrecernos alguna pista sobre la posibilidad de hacer extensibles buena parte de estas observaciones a su segunda filosofia, en la cual no dedicó ninguna atención a la ética. En realidad, son estos rasgos los que parecen estar detrás de la tesis (Iv) y (v). Planteémoslo en forma de interro- gante: ¿admitiría Wittgenstein, como hizo Kant al apelar a la estructura y la naturaleza de la Razón, que la tendencia hacia la metafísica y la ética son caracteristicas esenciales (universales y necesarias) del hombre $o$, por el contrario, serian propias nada más de algunos hombres pertenecientes a determinadas tradiciones culturales? $O$ incluso, tno se tratara de logros evolutivos o historicos de la especie humana $\rightarrow$ de algunos de sus miembros- y de esta manera, en tanto que simple «hecho empírico», explicables desde el mismo conocimiento de la realidad -por ejemplo, a partir de hechos psicológicos, culturales o económicospero bajo ningún sentido merecedores de un epiteto tan majestuoso como el de "trascendental"?

El primer Wittgenstein parece comprometerse con la que sería la tesis fuerte: (Iv) y (v) expresarian o, mejor, «mostrarían" rasgos "universales" y unecesarios» $\mathrm{y}$, por tanto, utrascendentales». Sin embargo, cuando Wittgenstein intenta explicitar el status o la naturaleza de (N) y (v) las cosas no parecen tan claras: por una parte, apela al hecho de que kel hombre tiene la tendencia a correr contra las barreras del lenguaje» $y$, por otra, a vivencias tales como «la admiración ante la existencia del mundos, ala vivencia de sentirse absolutamente seguro" y "la vivencia de sentirse culpable», las cuales parecen a veces ser la causa de buena parte de aquellas carreras. $Y$ el problema es que no parece claro que "vivencias" de esta suerte - y, por tanto, tampoco sus supuestos efectos- merezcan los calificativos de "universales», "necesarios" $y$ "trascendentales", y no ser tratadas como simples hechos psicologicos $y$ culturales. Nuevamente la indiferencia filosófica de Wittgenstein con respecto a los conocimientos positivos y científicos parece tener sus rendimientos. 
Aceptemos, no obstante, lo que Wittgenstein quiere que aceptemos -que en el Tractatus (IV) y (v) son "universales" y utrascendentales"- - y preguntemonos por la continuidad de su planteamiento. Aqui, sin duda, topamos con un problema hermenéutico: Wittgenstein guarda silencio sobre la ética: nihil ad rem. Podemos, con todo, hacer uso de sus observaciones sobre estética para obtener alguna luz. Así, en las lecciones que a estas cuestiones dedic 6 alrededor de 1938 encontramos un análisis del juicio estético (vid., p.e., pp. 33 y ss.) que podemos extrapolar mutatis mutandis al juicio ético. Quedaría corno sigue:

1) No existe la etica como disciplina filosófica, ya que carece de objeto propio, esto es, algo designado por los conceptos de "bueno" y «bien".

2) "Bueno" es un concepto que raramente acaece dentro de las actividades judicativas éticas. Por el contrario, su lugar natural parece ser el del aprendizaje lingüístico, y alli, solo con un valor "expresivo", un tipo de interjección equivalente a una señal de aprobación. Por otro lado, cuando "bueno" ocurre en el intercambio lingüístico ordinario no lo hace con la significación que la ética querría.

3) Por esta razón es necesario, al intentar caracterizar la etica, sustituir el concepto de "bueno" por el de «correcton.

4) "Correcto" reenvia a actividades lingürsticas donde existen "reglas" que fijan su significado, esto es, su "uso" en tanto que estas reglas ya suponen o establecen criterios para valorar o juzgar algo como "correcto".

5) En consecuencia, no hay usos absolutos del concepto "correcto" a ujuicios éticos" que sean "absolutos". Por contra, la práctica de juzgar se realiza sobre el trasfondo de instituciones judicativas y sólo dentro de ellas y solo relativamente a ellas tiene significación y aplicación el concepto "correcto".

Este análisis, según el propio Wittgenstein, es meramente un análisis descriptivo del tipo de práctica o de actividad lingüística en que consiste juzgar éticamente. Se trata de un análisis que no se basa en supuestas esencias de lo ético; tampoco en un subjetivismo o en un analisis causal que invoque los efectos de la acción; tampoco es un análisis que descanse en algún acto de reconocimiento de algo interior ni que apele a alguna instancia trascendente. Por el contrario, simplemente se trata de una reducción del juicio ético a una práctica lingúistica, según reglas. $Y$ en la medida en que estas reglas son relativas a las mismas prácticas que generan y dirigen, no existe nada parecido a juicios éticos absolutos. En Ética, al igual que en Estética, el concepto de "correcton es un asunto de reglas establecidas en la praxis social y en las costumbres y, por tanto, el relativismo parece inevitable.

Esta concepción del juicio ético es congruente con la concepción general que sobre el lenguaje mantuvo el segundo Wittgenstein. Siguiendo con nuestra extrapolación, existirían diversos juegos lingüísticos éticos "completos" en 51 mismos -entre los cuales es posible encontrar "parecidos de familia» $\sin$ tener todos ellos nada especial en común-y sólo dentro de cada uno de ellos, y en función de las reglas que los constituyen, tiene sentido hacer juicios de valor o de corrección. Con otras palabras, usamos el término kéticon para una gran variedad de sistemas que, evidentemente, poseen puntos en común, pero sin que ello implique que esos puntos en común sean la expresión de algo más fundamental o esencial.

De esta manera, para el segundo 
Wittgenstein «relativismo" y uantiesencialismo" en ética van de la mano: no solo los juicios éticos son relativos a un determinado sistema, sino que además no parece existir nada que pueda valer como una esencia de lo ético y que pudiese ser compartido. $Y$ es precisamente este antiesencialismo lo que acerca y separa al segundo Wittgenstein del primero. Lo acerca en tanto que continúa defendiendo la imposibilidad de la etica como un discurso justificador o crítico sobre la conducta de los hombres; lo separa en tanto que la nueva concepción del lenguaje le permite abandonar la trascendentalidad de la ética.

Ahora bien, ¿en qué medida esta retirada del trascendentalismo de la ética implicaba un cambio de postura existencial y ética de la persona llamada Wittgenstein? Claro está qư Wittgenstein no necesitaba abandonar su proyecto vital porque ya no pudiese reconocer a la ética como trascendental. En realidad, ahora sus propuestas parecen quedar en el lugar que les corresponde, pasando a ser un rasgo individual, aunque posiblemente recurrente y compartido por los hombres o por algunos de ellos. En concreto: ahora, aquello que enuncian las tesis (IV) y (v) se refiere a la manera como Ludwig Wittgenstein, qua individuo, se enfrentó al problema de su existencia $y$ resolvió los problemas que esta le planteaba. $\mathrm{Y}$ este era precisamente uno de los lugares adonde queríamos llegar: la continuidad de la actitud existencial básica de Wittgenstein que parece venir definida por el intento de resguardar o de proteger, mediante el ideal de la indiferencia, el yo o lo subjetivo -una de las divinidades wittgensteinianas- del mundo o de lo público - la otra divinidad. En este sentido, la continuidad de sus planteamientos existenciales y vitales parece que encontro expresion final en el balance que el mismo hizo de su vida, cuando en abril de 1951 en casa del Dr. Bevan le fue comunicada su inminente muerte: «Digales - le dijo a la Sra. Bevanque mi vida ha sido maravillosa».

Wittgenstein persiguio "la indiferenciaw como ideal y, como hemos visto, la justificó filosóficamente en tanto que para él era imposible un discurso explicativo y crítico de la conducta moral de los hombres. No parece, pues, que le falte razón a $\mathrm{H}$. Marcuse cuando en $E t$ hombre unidimensional, al analizar la sociedad de capitalismo avanzado, denuncia a la filosofía analítica y, en concreto, a la de Wittgenstein como la concepción de la filosofía que necesita una sociedad unidimensional para perpctuarse. La sociedad a la que ha dado origen el capitalismo avanzado, en opinión de Marcuse, se caracteriza por ser una sociedad en la que tanto la ideología como la tecnología devenida ideología están convirtiendo a la sociedad y al hombre en seres unidimensionales. Se trata de una reducción ideológica y vital importante: todo se agotaría en una única dimensión, la dimensión de lo patente, lo dado. En consecuencia, ya no cxiste una doblez que permita cuestionar el status quo. El hombre vive en una sola dimensión donde lo utópico, entendido como posibilidad de configurar lo social y lo vital de otra manera, se ha perdido. $Y$ en este marco la filosofía analítica $y$, en concreto, el proyecto de Wittgenstein representarian la renuncia y la miseria de la filosofía. En la medida en que la filosofia "debe dejar todo como esta", haciendo transparente e incuestionable «lo dado" estaría legitimando un estado de «conciencia infeliz» que se autorrepresenta a sí misma como "feliz".10

No podemos negar que le falte razón a Marcuse en su denuncia, digamos, "sociologican. Como ilustra nuestra tesis 
(III), Wittgenstein va en pos de la transparencia de lo dado, de la concepción cristalina de la realidad. Y a ello sólo habría que añadir la imposibilidad de la ética como discurso para entender que en Wittgenstein la filosofía no estaba llamada a transformar el mundo. Ahora bien, en descargo de Wittgenstein, podríamos decir que nada hay en su obra que permita colegir que el apostase por el carácter unidimensional del yo y del mundo; por el contrario, no era la suya una "conciencia feliz". Su apelación a "lo místico", su idea de la insatisfacción humana ante lo dado y su transparencia, su desagrado hacia el cientificismo y tecnologicismo de la época en que vivio, su propuesta vital, etc., no parecen convertirlo en un hombre unidimensional. Distinto es que Wittgenstein creyese que el mundo y la sociedad fuesen tan racionales como para permitir un discurso y una acción encaminada hacia la realización de lo utópico. Su propuesta vital es individualista, producto de su profundo escepticismo no sólo hacia los supuestos beneficios vitales del cientificismo y de la forma de vida cientifica, sino también hacia la posibilidad de una solución colectiva de los problemas humanos. No se trataba de transformar el mundo sino de modificar, dejando de lado extravios metafísicos, nuestra manera de vivenciarlo. Y no habria posibilidad de transfommar el mundo porque el mundo no era, para Wittgenstein, totalmente racional. Su pensamiento establece un hiato insalvable entre el ambito de lo racional, que es el ámbito del conocimiento y del discurso, y ese fondo irracional al que pertenecerfa la felicidad que, siendo lo más importante, quedaba fuera del discurso $y$ de la acción social. Como se sugirió más arriba, tras el caldero tiró la cuerda.
1. W. Warren Bartley III, Witgenstein, Madrid, Cátedra, 1982.

2. P. Engelmann, Letters from Ludwig Witgenstein, Oxford, Basil Blackwell, 1967, p. 35.

3. A. Janik y S. Toulmin, La Viena de Wittgenstein, Madrid, Taurus, 1974.

4. W. James, Pragmatismo, Buenos Aires, Aguilar, 1975, pp. 25 y ss.

5. Lao-Tsé, Tao Te Ching (trad. de Chú Ta-Kao y Caridad Draz Faes), Madrid, Morata, 1961, XLIII, p. 72 .
6. R. Camap, «a superación de la metafísica mediante el analisis logico del lenguajen (1932), en A.J. Ayer (ed.), El postlivismo logico, Madrid, FCE, 1965.

7. P.F. Strawson, Individuals, Londres, Methuen, 1959, pp. 99 y ss.

8. F. Waismann L. Wittgenstein y el Circulo de Viena (1967), Mexico, FCE, 1973.

9. J. Ferrater Mora, De la materia a la razón, Madrid, Alianza, 1979, pp. 152 y $\mathrm{ss.}$

10. H. Marcuse, El hombre unidintensiontal (1954), Barcelona, Seix Barral, 1968. pp, 156 y ss.

\section{BIBLIOGRAFIA}

WITTGenstein, L.: Tractatus logico-philosophicus (trad. de Jacobo Muñoz y I. Reguera), Madrid, Alianza, 1987.

-: Investigaciontes filosoficas (trad. de A.G. Suarez y U. Moulines), Barcelona, Crítica, 1988.

-: Notebooks (ed. de G.H. von Wright y G.E.M. Anscombe; trad. de G.E.M. Anscombe), Oxford, Basil Blackwell, 1969.

- Observaciones sobre La Rana Dorada de Frazer (trad, de J. Sádaba), Madrid, Tecnos, 1992.

$\rightarrow$ Philosophical Grammar (Philosophische Grant- matik) (ed. de $\mathrm{R}$. Rhees; trad. inglesa de $\mathbf{A}$. Kenny), Oxford, Basil Blackwell, 1974.

-: Cultwe and Vahe (Vernischte Benterkungen) (ed. de G.H. von Wright; trad. ingl. de P. Winch), Oxford, Basil Blackwell, 1980.

-: Conferencia sobre Ética (trad, de F. Birulés), Barcelona, Paidós / L.C.E.-U.A.B., 1989.

-: Lectures and Conversations on Aesthetics. Psychology and Religious Belief (ed. de C. Barret), Oxford, Basil Blackwell, 1966. 